\title{
CONTACT PROCESS WITH DESTRUCTION OF CUBES AND HYPERPLANES: FOREST FIRES VERSUS TORNADOES
}

\author{
N. LANCHIER, * Arizona State University
}

\begin{abstract}
Nonspatial stochastic models of populations subject to catastrophic events result in the common conclusion that the survival probability of the population is nondecreasing with respect to the random number of individuals removed at each catastrophe. The purpose of this paper is to prove that such a monotonic relationship is not true for simple spatial models based on Harris' contact processes, whose dynamics are described by hypergraph structures rather than traditional graph structures. More precisely, it is proved that, for a wide range of parameters, the destruction of (infinite) hyperplanes does not affect the existence of a nontrivial invariant measure, whereas the destruction of large (finite) cubes drives the population to extinction, a result that we depict by using the biological picture: forest fires are more devastating than tornadoes. This indicates that the geometry of the subsets struck by catastrophes is somewhat more important than their area, thus the need to consider spatial rather than nonspatial models in this context.
\end{abstract}

Keywords: Contact process; catastrophe; hypergraph; forest fire; tornado

2010 Mathematics Subject Classification: Primary 60K35

\section{Introduction}

Communities subject to the effect of catastrophic events resulting in the removal of all or part of the individuals are ubiquitous in nature, and a number of stochastic models for the growth of such populations have been extensively studied in the literature. The simplest and generic model that describes these dynamics is the simple birth-and-death process supplemented with so-called binomial catastrophes. In the absence of catastrophes, individuals give birth independently of each other to a new individual at rate $\alpha$ and die at rate 1 . Therefore, the system only experiences individual deaths as individuals are removed one at a time. Including binomial catastrophes, individuals are in addition simultaneously exposed to catastrophic events occurring at rate $\Lambda$ and, independently of each other, survive the catastrophe with probability $p$, which induces transition rates that take the form of the binomial distribution. More precisely, letting $X_{t}$ denote the number of individuals or population size at time $t$, the dynamics are formally described by the Markov generator

$$
\begin{aligned}
\lim _{h \rightarrow 0} h^{-1}\left[\mathrm{E} f\left(X_{t+h}\right)-\mathrm{E} f\left(X_{t}\right)\right]= & \alpha X_{t}\left[f\left(X_{t}+1\right)-f\left(X_{t}\right)\right]+X_{t}\left[f\left(X_{t}-1\right)-f\left(X_{t}\right)\right] \\
& +\Lambda \sum_{X=0}^{X_{t}}\left(\begin{array}{c}
X_{t} \\
X
\end{array}\right) p^{X}(1-p)^{X_{t}-X}\left[f(X)-f\left(X_{t}\right)\right] .
\end{aligned}
$$

Received 19 July 2010; revision received 1 February 2011.

* Postal address: School of Mathematical and Statistical Sciences, Arizona State University, Tempe, AZ 85287, USA.

Email address: lanchier@math.asu.edu

Research supported in part by NSF grant DMS-10-05282. 
Even though a wide variety of stochastic models have been developed in order to understand the effect of random catastrophic events on the survival probability of a population, most of them, similarly to the generic model (1), exclude the presence of a spatial structure, i.e. the state space of these stochastic processes keeps track of a number of individuals but ignores the spatial arrangement of the population. This leads unavoidably to the following conclusion, which directly follows from standard coupling arguments: increasing stochastically the random number of individuals removed at each disaster given the population size can only decrease the survival probability. The main objective of this paper is to prove that the previous conclusion does not hold for very simple spatially explicit models based on Harris' contact process [6] which we express, in the spatial context, as a nonmonotonic relationship between the number of lattice sites struck by each catastrophic event and the survival probability of the process.

Note that the inclusion of catastrophic events that hit each lattice site individually at a constant rate in the contact process results in another contact process with a larger death rate; therefore, the process survives whenever the birth rate is sufficiently large. At the other extreme, if catastrophic events hit the whole lattice, thus consisting of the simultaneous removal of all individuals, then the process starting from any configuration dies out almost surely in a finite time regardless of the birth rate. This reveals an obvious lack of monotonicity between the survival probability and the rate at which each individual is struck by a catastrophic event, which holds for both spatial and nonspatial models. The two examples above also suggest that there is a monotonic relationship between the survival probability and the number of lattice sites (a single site versus the whole lattice) struck by each catastrophic event. We prove, however, that, with the birth rate, the individual death rate, and the rate at which each individual is hit by a catastrophic event being fixed, the inclusion of catastrophic events removing all individuals in infinite subsets of the spatial structure may preserve the existence of a nontrivial invariant measure, whereas the removal of all individuals in finite subsets may drive the process to an almost-sure extinction. This somewhat surprising result contrasts drastically with the predictions based on nonspatial models, which shows the importance of considering models that include a spatial structure. The key to our proof is to compare models in which subsets struck by catastrophes have different geometrical shapes, a component that cannot be incorporated into nonspatial models since they do not include any geometrical structure. On the other hand, though there are several other examples of stochastic processes including both a spatial structure and catastrophic events [1], [2], [5], [7], [9], [11], [12], [13], these models are neither designed to understand the impact of the geometry of the catastrophes on the survival probability of the population, nor to support the existence of a nonmonotonic relationship between the area of the subsets struck by each catastrophic event and the survival probability.

\subsection{Description of the models}

In the simple birth-and-death process, individuals give birth to a new individual at rate $\alpha$ and die at rate 1 . To include a spatial structure, a natural approach is to assume that individuals are located on the $d$-dimensional regular lattice $\mathbb{Z}^{d}$, with each lattice site being either empty or occupied by one individual, and that each offspring is sent uniformly at random to one of the $2 d$ nearest neighbors of its parent's site. In addition, individuals sent to a site which is already occupied are instantaneously removed from the system in order to model competition for space. The resulting process is Harris' contact process [6]. The state space consists of all the functions that map the set of lattice sites into $\{0,1\}$, where state 0 means empty and state 1 
means occupied, and the dynamics are described by the Markov generator

$$
L f(\zeta)=\frac{\alpha}{2 d} \sum_{x \in \mathbb{Z}^{d}} \sum_{y \sim x} \zeta(y)\left[f\left(\zeta_{x, 1}\right)-f(\zeta)\right]+\sum_{x \in \mathbb{Z}^{d}}\left[f\left(\zeta_{x, 0}\right)-f(\zeta)\right],
$$

where $\zeta_{x, i}$ is the configuration obtained from $\zeta$ by assigning the value $i$ to vertex $x$, and where we use the notation $y \sim x$ to indicate that vertices $x$ and $y$ are Euclidean distance 1 apart. Thinking of the regular lattice as a graph in which edges connect nearest neighbors, the expression of the generator indicates that deaths occur independently at each vertex while births occur independently through the edges. Now, it is interesting to think of contact processes including catastrophic events as being induced by a hypergraph (rather than a traditional graph) structure. That is, we complete the regular lattice with a collection of hyperedges, i.e. subsets of the vertex set with arbitrary cardinality, which results in a hypergraph. Then, we assume that catastrophic events consist of the removal of all the individuals in the same hyperedge, so there are three types of event corresponding to three types of object of the hypergraph: death events attached to each vertex, birth events attached to each (oriented) edge, and catastrophic events attached to each hyperedge. Since our purpose is to prove that the survival probability is not monotonically related to the size of the catastrophic events (cardinality of the hyperedges), for simplicity, we focus only on two special cases of hypergraphs. In the first case, the set of hyperedges consists of the hyperplanes

$$
\mathcal{T}_{x}=\left\{\left(x_{1}, x_{2}, \ldots, x_{d}\right) \in \mathbb{Z}^{d}: x_{1}=x\right\}, \quad x \in \mathbb{Z},
$$

while in the second case, it consists of the set of $d$-dimensional cubes

$$
\mathcal{F}_{x}=\left\{y \in \mathbb{Z}^{d}: \max \left\{\left|x_{1}-y_{1}\right|, \ldots,\left|x_{d}-y_{d}\right|\right\} \leq K\right\}, \quad x \in \mathbb{Z}^{d},
$$

where $K$ is a large integer. The two stochastic models we consider have the same state space as, and are naturally derived from, the contact process (2), but include in addition the removal at some specific rates of all the individuals simultaneously present either in the same hyperplane or in the same cube. Formally, the dynamics of the first process are described by

$$
\begin{aligned}
L_{T} f(\xi)= & \frac{\alpha}{2 d} \sum_{x \in \mathbb{Z}^{d}} \sum_{y \sim x} \xi(y)\left[f\left(\xi_{x, 1}\right)-f(\xi)\right]+\sum_{x \in \mathbb{Z}^{d}}\left[f\left(\xi_{x, 0}\right)-f(\xi)\right] \\
& +\Lambda \sum_{x \in \mathbb{Z}}\left[f\left(\xi_{\mathcal{T}_{x}, 0}\right)-f(\xi)\right],
\end{aligned}
$$

where, for each $\mathcal{B} \subset \mathbb{Z}^{d}$, the configuration $\xi_{\mathcal{B}, 0}$ is obtained from $\xi$ by assigning the value 0 to all the vertices belonging to the hyperedge $\mathcal{B}$, while the dynamics of the second process are given by

$$
\begin{aligned}
L_{F} f(\eta)= & \frac{\alpha}{2 d} \sum_{x \in \mathbb{Z}^{d}} \sum_{y \sim x} \eta(y)\left[f\left(\eta_{x, 1}\right)-f(\eta)\right]+\sum_{x \in \mathbb{Z}^{d}}\left[f\left(\eta_{x, 0}\right)-f(\eta)\right] \\
& +\Lambda(2 K+1)^{-d} \sum_{x \in \mathbb{Z}^{d}}\left[f\left(\eta_{\mathcal{F}_{x}, 0}\right)-f(\eta)\right]
\end{aligned}
$$

where configuration $\eta_{\mathcal{B}, 0}$ is obtained from $\eta$ in the same manner. Thinking of the twodimensional case, we call the stochastic model in (3) the contact process with tornadoes, while we call the stochastic model in (4) the contact process with forest fires (see Figure 1 

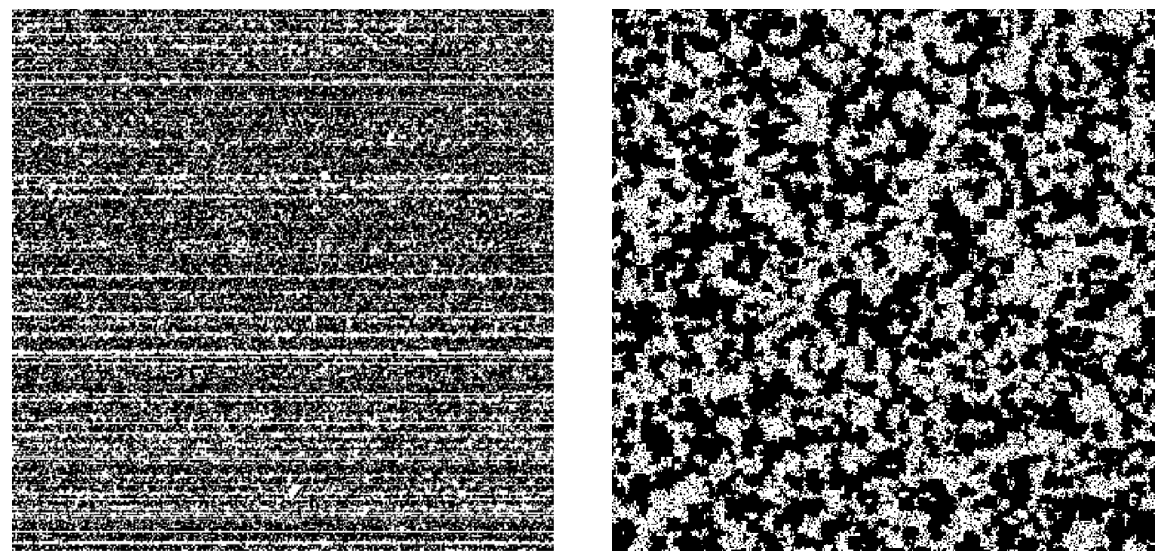

Figure 1: Pictures of the quasistationary equilibrium of the contact process with tornadoes $(l e f t)$ and the contact process with forest fires (right) in the case of nearest-neighbor interactions on a $400 \times 400$ lattice. In both pictures, the parameters are $\alpha=5$ and $\Lambda=\frac{1}{2}$, and the range of a fire is $2 K+1=9$.

for simulation pictures). This terminology is motivated by the fact that strong tornadoes are typically $1 \mathrm{~km}$ across but stay on the ground for more than $100 \mathrm{~km}$, whereas forest fires start at a specific point and basically spread out at the same speed in each direction. Although, for simplicity, the geometric shapes we are considering are somewhat idealized, we point out that our results for the contact process with tornadoes easily extend to hyperedges which are basically unbounded $(d-1)$-dimensional objects, while our results for the contact process with forest fires extend to hyperedges which are large bounded $d$-dimensional objects. We note from the generators (3) and (4) that tornadoes occur in each hyperplane at rate $\Lambda$, whereas in the process with forest fires this rate is divided by the number of vertices destroyed by each fire. This is motivated by the fact that each lattice site is contained in one and only one hyperplane $\mathcal{T}_{x}$, whereas it is contained in exactly $(2 K+1)^{d}$ cubes $\mathcal{F}_{x}$. The rate is thus rescaled in such a way that, for both processes, each individual is removed due to a catastrophic event at the same rate $\Lambda$. In particular, the nonspatial mean-field approximations of both processes are equal and described by the differential equation

$$
\frac{\mathrm{d} u}{\mathrm{~d} t}=\alpha u(1-u)-(1+\Lambda) u,
$$

where the function $u(t)$ denotes the population density at time $t$. Therefore, we can emphasize the fact that any disagreement between the process with tornadoes and the process with forest fires is indeed due to the geometric structure of the underlying hypergraphs rather than an inappropriate choice of the parameters of the processes.

\subsection{Tornadoes versus forest fires}

The nonmonotonic relationship between the size of the catastrophic events (cardinality of the hyperedges) and the survival probability of the processes are expressed in terms of critical values. Note that standard coupling arguments imply that the survival probability is nondecreasing with respect to the birth rate $\alpha$. This induces the existence of a unique phase transition; hence, for 
each $\Lambda \geq 0$ and $K \in \mathbb{Z}_{+}$, we introduce the critical values

$$
\begin{aligned}
\alpha_{\mathrm{c}}(\xi, \Lambda) & =\sup \left\{\alpha: \mathrm{P}\left(\xi_{t} \neq \varnothing \text { for all } t>0 \mid \xi_{0}=\{0\}\right)=0\right\}, \\
\alpha_{\mathrm{c}}(\eta, K, \Lambda) & =\sup \left\{\alpha: \mathrm{P}\left(\eta_{t} \neq \varnothing \text { for all } t>0 \mid \eta_{0}=\{0\}\right)=0\right\},
\end{aligned}
$$

where processes are identified with the set of occupied sites. Our first two results state that the contact process with tornadoes survives for reasonably large values of the birth rate, whereas, for any choice of the birth parameter, the contact process with forest fires dies out exponentially fast whenever the scale parameter $K$ is sufficiently large.

Theorem 1. (Tornadoes.) We have $\alpha_{\mathrm{c}}(\xi, \Lambda) \leq 2 d(1+\Lambda)$ and $\lim _{\Lambda \rightarrow 0} \alpha_{\mathrm{c}}(\xi, \Lambda)=\alpha_{\mathrm{c}}(\xi, 0)$.

Theorem 2. (Forest fires.) For all $\Lambda>0, \lim _{K \rightarrow \infty} \alpha_{\mathrm{c}}(\eta, K, \Lambda)=\infty$.

Theorems 1 and 2 indicate that, at least for sufficiently large $K$, there is a wide range of birth rates for which the inclusion of tornadoes does not affect the existence of a nontrivial invariant measure, whereas introducing forest fires drives the process to extinction. On the other hand, starting from the configuration in which all sites are occupied, it can be proved that each tornado removes infinitely many individuals, while each forest fire obviously removes only finitely many individuals. The intuition behind these results and their proofs is that the graph distance in $\mathbb{Z}^{d}$ between the complement of a hyperplane and any of its vertices is equal to 1 , whereas the graph distance between the complement of a cube and its center can be made arbitrarily large. Thinking of the graph distance as a number of births in the nearest-neighbor case, this indicates that, though infinite, hyperplanes are repopulated somewhat faster than large cubes. Note however that this property is no longer true when the range of the interactions increases up to the range of the forest fires, in which case we prove that the distinction between tornadoes and forest fires is weakened. Formally, the processes with long-range interactions are obtained from the generators (3) and (4) by assuming that individuals evolve on the rescaled lattice $\mathbb{Z}^{d} / N$, taking the sum over all vertices $y$ within distance 1 of vertex $x$, and renormalizing the sum by the new neighborhood size. More precisely, the Markov generator of the long-range contact process with tornadoes is

$$
\begin{aligned}
L_{T} f(\xi)= & \alpha(2 N+1)^{-d} \sum_{x \in \mathbb{Z}^{d} / N} \sum_{\|x-y\| \leq 1} \xi(y)\left[f\left(\xi_{x, 1}\right)-f(\xi)\right] \\
& +\sum_{x \in \mathbb{Z}^{d} / N}\left[f\left(\xi_{x, 0}\right)-f(\xi)\right]+\Lambda \sum_{x \in \mathbb{Z} / N}\left[f\left(\xi_{\mathcal{T}_{x}, 0}\right)-f(\xi)\right],
\end{aligned}
$$

where $\|x-y\|=\max \left\{\left|x_{1}-y_{1}\right|, \ldots,\left|x_{d}-y_{d}\right|\right\}$ and

$$
\mathcal{T}_{x}=\left\{\left(x_{1}, x_{2}, \ldots, x_{d}\right) \in \frac{\mathbb{Z}^{d}}{N}: x_{1}=x\right\}, \quad x \in \mathbb{Z} / N .
$$

The long-range contact process with forest fires is obtained similarly, except that cubes are also rescaled by a factor $N$, which gives the Markov generator

$$
\begin{aligned}
L_{F} f(\eta)= & \alpha(2 N+1)^{-d} \sum_{x \in \mathbb{Z}^{d} / N} \sum_{\|x-y\| \leq 1} \eta(y)\left[f\left(\eta_{x, 1}\right)-f(\eta)\right] \\
& +\sum_{x \in \mathbb{Z}^{d} / N}\left[f\left(\eta_{x, 0}\right)-f(\eta)\right]+\Lambda(2 K+1)^{-d} \sum_{x \in \mathbb{Z}^{d} / N}\left[f\left(\eta_{\mathcal{F}_{x}, 0}\right)-f(\eta)\right],
\end{aligned}
$$



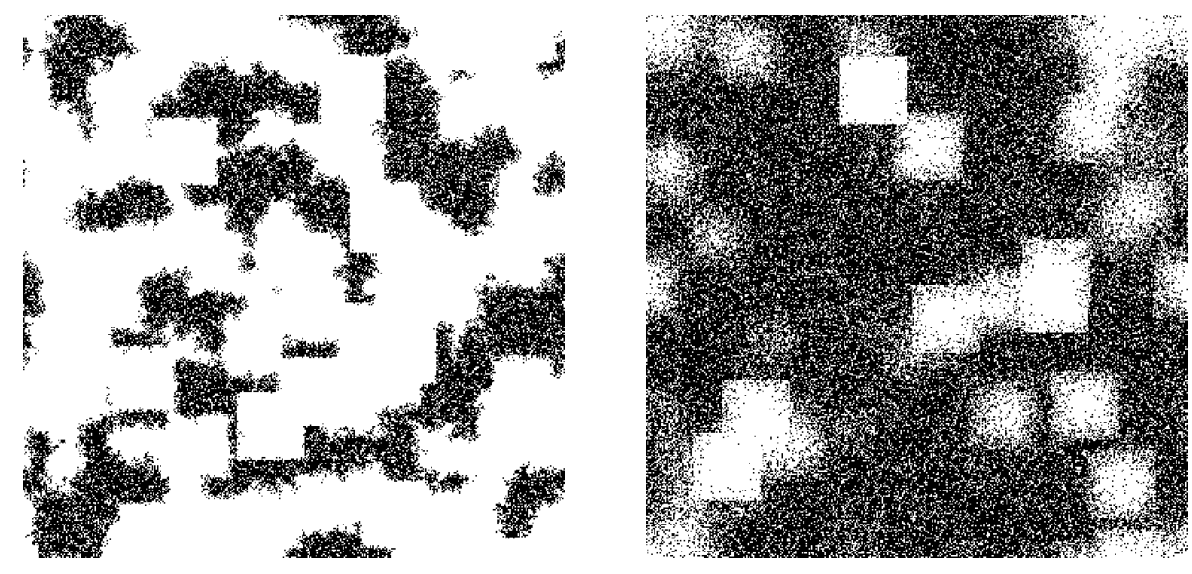

Figure 2: Pictures of the quasistationary equilibrium of the process with forest fires. In both simulations, the parameters are $\alpha=5, \Lambda=\frac{1}{2}$, and $2 K+1=49$. The dispersal range is equal to 2 and 10 , respectively.

where we now have

$$
\mathcal{F}_{x}=\left\{y \in \frac{\mathbb{Z}^{d}}{N}: \max \left\{\left|x_{1}-y_{1}\right|, \ldots,\left|x_{d}-y_{d}\right|\right\} \leq \frac{K}{N}\right\}, \quad x \in \mathbb{Z}^{d} / N .
$$

See Figure 2 for simulation pictures of the process with forest fires with dispersal range 2 and 10 , respectively. The long-term behavior in the limit as the range of the interactions $N$ tends to $\infty$ is then described by the following two theorems.

Theorem 3. (Tornadoes.) We have $\lim _{N \rightarrow \infty} \alpha_{\mathrm{c}}(\xi, \Lambda)=1+\Lambda$.

Theorem 4. (Forest fires.) For all $K>0, \lim _{N \rightarrow \infty} \alpha_{\mathrm{c}}(\eta, K, \Lambda)=1+\Lambda$.

Theorems 3 and 4 indicate that, for a very large range of interactions, both the contact process with tornadoes and the contact process with forest fires result in the same predictions as their common mean-field model (5) in terms of the convergence of their respective critical values to the critical value of the mean-field approximation. This shows that the geometry of the subsets struck by catastrophes, which is an important component in the case of short-range interactions, becomes less and less relevant as the dispersal range increases. The key to proving these two convergence results is to establish weak convergence of the contact processes to the same branching random walk with birth rate $\alpha$ and death rate $1+\Lambda$.

\section{Proof of Theorem 1 (tornadoes)}

Let $\Gamma$ be the orthogonal complement of $\mathcal{T}_{0}$, and let $\tilde{\xi}_{t}(\alpha, \Lambda)$ be the contact process with tornadoes modified so that particles sent outside the straight line $\Gamma$ are killed. Noting that

1. at each birth, the new offspring is sent to a site in $\Gamma$ with probability $1 / d$, and

2. for all $x \in \mathbb{Z}$, the hyperplane $\mathcal{T}_{x}$ intersects the straight line $\Gamma$ at exactly one site

we find that the modified process $\tilde{\xi}_{t}(\alpha, \Lambda)$ is the one-dimensional basic contact process in which particles give birth at rate $\alpha / d$ and die at rate $1+\Lambda$. By construction, the contact process with 
tornadoes dominates stochastically this basic contact process, which implies that

$$
\alpha_{\mathrm{c}}(\xi, \Lambda) \leq d(1+\Lambda) \alpha_{\mathrm{c}}(d=1) \leq 2 d(1+\Lambda),
$$

where $\alpha_{\mathrm{c}}(d=1)$ is the critical value of the one-dimensional basic contact process with normalized death parameter equal to 1 . In particular, the last inequality follows from the fact that this critical value is less than 2 , a result that can be found in [8, p. 43]. This completes the proof of the first statement of Theorem 1.

Turning to the second statement, we first observe that the monotonicity of the survival probability with respect to the parameter $\Lambda$ directly implies that

$$
\alpha_{\mathrm{c}}(\xi, \Lambda) \geq \alpha_{\mathrm{c}}(\xi, 0) \quad \text { for all } \Lambda \geq 0 ; \quad \text { therefore, } \quad \liminf _{\Lambda \rightarrow 0} \alpha_{\mathrm{c}}(\xi, \Lambda) \geq \alpha_{\mathrm{c}}(\xi, 0) .
$$

To show the reverse inequality, we fix $\alpha>\alpha_{\mathrm{c}}(\xi, 0)$, the critical value of the $d$-dimensional basic contact process, and, given $L, T \in \mathbb{Z}_{+}$define the spatial and space-time regions

$$
\begin{gathered}
D_{z}=2 L z+[-L, L]^{d} \quad \text { for all } z \in \mathbb{Z} \\
B_{z, n}=\left(2 L z+[-4 L, 4 L]^{d}\right) \times(n T,(n+1) T) \text { for all }(z, n) \in \mathbb{Z} \times \mathbb{Z}_{+} .
\end{gathered}
$$

Then, for all $\varepsilon>0$, the space and time scales $L$ and $T$ can be chosen in such a way that

$$
\mathrm{P}\left(\operatorname{card}\left(\xi_{(n+1) T}(\alpha, 0) \cap D_{z \pm 1}\right) \geq M \mid \operatorname{card}\left(\xi_{n T}(\alpha, 0) \cap D_{z}\right) \geq M\right) \geq 1-\varepsilon
$$

for a suitable $M>0$. In addition, this holds for the basic contact process modified so that particles sent outside the space-time box $B_{z, n}$ are killed. We refer the reader to [3] for a proof of this statement. In particular, property (6) can be extended to the contact process with tornadoes provided that $\Lambda>0$ is sufficiently small. Indeed, the space and time scales being fixed, the probability of a tornado occurring in the space-time box $B_{z, n}$ is bounded by

$$
1-\exp (-8 L T \Lambda) \leq \varepsilon \text { for all } \Lambda \leq \Lambda(L, T, \varepsilon) .
$$

It follows that, for all $\alpha>\alpha_{\mathrm{c}}(\xi, 0)$ and all $\Lambda \leq \Lambda(L, T, \varepsilon)$,

$$
\mathrm{P}\left(\operatorname{card}\left(\xi_{(n+1) T}(\alpha, \Lambda) \cap D_{z \pm 1}\right) \geq M \mid \operatorname{card}\left(\xi_{n T}(\alpha, \Lambda) \cap D_{z}\right) \geq M\right) \geq 1-2 \varepsilon .
$$

Positive probability of survival of the contact process with tornadoes for such parameters can be deduced from (7) by choosing $\varepsilon>0$ small and by using standard coupling arguments to compare the process with one-dependent oriented site percolation, and we again refer the reader to [3] for a proof of this statement. In conclusion, if the process in the absence of tornadoes survives then it does in the presence of tornadoes provided that $\Lambda>0$ is sufficiently small, i.e.

$$
\alpha>\alpha_{\mathrm{c}}(\xi, 0) \text { implies that } \alpha>\alpha_{\mathrm{c}}(\xi, \Lambda) \text { for } \Lambda>0 \text { small, }
$$

which is equivalent to $\lim \sup _{\Lambda \rightarrow 0} \alpha_{\mathrm{c}}(\xi, \Lambda) \leq \alpha_{\mathrm{c}}(\xi, 0)$. This completes the proof.

\section{Proof of Theorem 2 (forest fires)}

Note that Theorem 2 is equivalent to the statement: for all $\alpha<\infty$ (even large) and all $\Lambda>0$ (even small), the contact process converges almost surely to the 'all empty' configuration whenever the size of a fire $K$ is sufficiently large, which we prove in this section. The key idea 
is that, regardless of the initial configuration, any given cube of fixed size, say $A$, will be empty at a certain deterministic time with a probability that approaches 1 as $K$ tends to $\infty$. This follows from a two-step reasoning: (i) when $K$ is large, we can find a large cube containing $A$, say $B$, that is quickly cleared by a catastrophic event, then (ii) even in the absence of deaths, either natural or due to a catastrophe, once $B$ is cleared, the time for the particles to repopulate $A$ is large. We prove these two statements in the next two lemmas in reverse order. Property (ii) is true even in the absence of deaths so, for simplicity, we show the result for the Richardson process [10] with birth parameter $\alpha$, a process that we denote by $\Theta_{t}(\alpha)$. Later on, we also add, when it is relevant, a superscript to indicate the initial configuration of the processes under consideration, with the superscript 0 meaning that there is initially a single particle at the origin.

Lemma 1. For $T \in \mathbb{Z}_{+}$and $a, b>0$, we introduce the sets

$$
A=(-a T, a T)^{d} \text { and } B=(-8 b T, 8 b T)^{d} .
$$

There exist $a \leq b$ depending only on $\alpha$ such that, for all $\varepsilon>0$,

$$
\mathrm{P}\left(\Theta_{T}^{B^{\mathrm{c}}}(\alpha) \cap A \neq \varnothing\right) \leq \frac{1}{2} \varepsilon \quad \text { for all sufficiently large } T .
$$

Proof. We start by applying the shape theorem for the Richardson process to obtain the existence of a convex set $H$ with nonempty interior such that, for all $\varepsilon>0$,

$$
\mathrm{P}\left(H \subset t^{-1} \Theta_{t}^{0}(\alpha) \subset 2 H\right)>1-\frac{1}{4} \varepsilon \text { for sufficiently large } t .
$$

We will prove the lemma for the constants

$$
a:=\inf _{x \in \partial H}\|x\|_{\infty} \quad \text { and } \quad b:=\sup _{x \in \partial H}\|x\|_{\infty},
$$

where $\partial H$ denotes the topological boundary of the convex set $H$. By applying twice (the weak version of) the shape theorem given by (8), we obtain

$$
\begin{aligned}
\mathrm{P}\left(\Theta_{T}^{A}(\alpha) \cap B^{\mathrm{c}} \neq \varnothing\right) & =\mathrm{P}\left(\Theta_{T}^{A}(\alpha) \not \subset B\right) \\
& \leq \mathrm{P}\left(\Theta_{2 T}^{0}(\alpha) \not \subset B \mid \Theta_{T}^{0}(\alpha) \supset A\right) \\
& \leq \frac{\mathrm{P}\left(\Theta_{2 T}^{0}(\alpha) \not \subset B\right)}{\mathrm{P}\left(\Theta_{T}^{0}(\alpha) \supset A\right)} \\
& \leq \frac{\mathrm{P}\left(\Theta_{2 T}^{0}(\alpha) \not \subset 8 T H\right)}{\mathrm{P}\left(\Theta_{T}^{0}(\alpha) \supset T H\right)} \\
& \leq \frac{\mathrm{P}\left((2 T)^{-1} \Theta_{2 T}^{0}(\alpha) \not \subset 4 H\right)}{\mathrm{P}\left(T^{-1} \Theta_{T}^{0}(\alpha) \supset H\right)} \\
& \leq\left(\frac{1}{4} \varepsilon\right)\left(1-\frac{1}{4} \varepsilon\right)^{-1} \\
& \leq \frac{1}{2} \varepsilon
\end{aligned}
$$

for all sufficiently large $T$ since $8 T H \subset B$ and $A \subset T H$. Finally, by invoking the fact that the Richardson process is self-dual, we can conclude that

$$
\mathrm{P}\left(\Theta_{T}^{B^{\mathrm{c}}}(\alpha) \cap A \neq \varnothing\right)=\mathrm{P}\left(\Theta_{T}^{A}(\alpha) \cap B^{\mathrm{c}} \neq \varnothing\right) \leq \frac{1}{2} \varepsilon
$$

for all sufficiently large $T$. This completes the proof. 
The proof of property (i) along with its combination with (ii) that leads to Theorem 2 are given in the next lemma. To state the latter, we introduce,

$$
A_{x, j}=(a T x+A) \times\left(\frac{1}{2} j T+\left(0, \frac{1}{2} T\right)\right) \text { for all }(x, j) \in \mathbb{Z}^{d} \times \mathbb{Z}_{+}
$$

with $a=a(\alpha)$ and $A$ defined as in Lemma 1 , and declare site $(x, j)$ to be open whenever the corresponding space-time box $A_{x, j}$ is empty for the contact process with catastrophes.

Lemma 2. Assume that $\Lambda>0$. For all $\varepsilon>0$, there exist $T$ and $K(\varepsilon)$ such that

$$
\mathrm{P}((x, j) \text { is open }) \geq 1-\varepsilon \text { for all }(x, j) \in \mathbb{Z}^{d} \times \mathbb{Z}_{+}^{*} \text { and } K \geq K(\varepsilon) .
$$

Proof. Since the evolution rules are translation invariant in space and time, and the contact process is attractive, it suffices to prove the result for site $(x, j)=(0,1)$ and when all lattice sites are initially occupied, which is equivalent to

$$
\mathrm{P}\left(\eta_{t}(\alpha, \Lambda) \cap A \neq \varnothing \text { for some } t \in\left(\frac{1}{2} T, T\right)\right) \leq \varepsilon \quad \text { for large } K .
$$

We prove (9) for large $T$ and all $K \geq 16 b T$, where $b=b(\alpha)$ is defined as in Lemma 1 . Since each $K$-cube centered at a site within distance $K / 2$ of the origin contains the set $B$, introducing independent exponential random variables $X_{i}$ with parameter $\Lambda(2 K+1)^{-d}$, we have

$$
\begin{aligned}
\mathrm{P}\left(\eta_{t}(\alpha, \Lambda) \cap B \neq \varnothing \text { for all } t \in\left(0, \frac{1}{2} T\right)\right) & \leq \prod_{i=1}^{(K-1)^{d}} \mathrm{P}\left(X_{i}>\frac{1}{2} T\right) \\
& \leq \prod_{i=1}^{(K-1)^{d}} \exp \left(-\frac{1}{2} \Lambda(2 K+1)^{-d} T\right) \\
& \leq \exp \left(-\frac{\Lambda T}{2^{d+2}}\right) \\
& \leq \frac{1}{2} \varepsilon
\end{aligned}
$$

for large $T$ since each $K$-cube is cleared by a fire at rate $\Lambda(2 K+1)^{-d}$. Using the facts that the Richardson model dominates stochastically the contact process, can only increase as a pure birth process, and is attractive, and applying Lemma 1, we also have

$$
\begin{aligned}
\mathrm{P}\left(\eta_{t}\right. & \left.\cap A \neq \varnothing \text { for some } t \in\left(\frac{1}{2} T, T\right) \mid \eta_{t} \cap B=\varnothing \text { for some } t<\frac{1}{2} T\right) \\
& \leq \mathrm{P}\left(\Theta_{t} \cap A \neq \varnothing \text { for some } t \in\left(\frac{1}{2} T, T\right) \mid \Theta_{t} \cap B=\varnothing \text { for some } t<\frac{1}{2} T\right) \\
& \leq \mathrm{P}\left(\Theta_{t} \cap A \neq \varnothing \text { for some } t \in\left(\frac{1}{2} T, T\right) \mid \Theta_{0} \cap B=\varnothing\right) \\
& \leq \mathrm{P}\left(\Theta_{t} \cap A \neq \varnothing \text { for some } t \in\left(\frac{1}{2} T, T\right) \mid \Theta_{0}=B^{\mathrm{c}}\right) \\
& \leq \mathrm{P}\left(\Theta_{T}^{B^{\mathrm{c}}} \cap A \neq \varnothing\right) \\
& \leq \frac{1}{2} \varepsilon
\end{aligned}
$$

for all large $T$. Combining (10) and (11), we obtain (9). The lemma follows.

To complete the proof of Theorem 2, the idea is to couple the contact process properly rescaled in space and time with a certain oriented site percolation process. Let $\mathscr{H}$ be the 
directed graph with vertex set $\mathbb{Z}^{d} \times \mathbb{Z}_{+}$in which there is an edge directed from $(x, i)$ to $(y, j)$ if and only if

$$
\|x-y\|=1 \quad \text { and } \quad i=j \quad \text { or } \quad x=y \quad \text { and } \quad j=i+1 .
$$

Lemma 2 indicates that, for all $\varepsilon>0$, parameters can be chosen in such a way that the set of open sites for the contact process dominates stochastically the set of open sites in the oriented site percolation process with parameter $1-\varepsilon$ on the directed graph $\mathscr{H}$. For small $\varepsilon>0$, the probability of a path of length $n$ of closed sites in the percolation process decreases exponentially fast with $n$, as proved in [14, Inequality (8.2)]. Since the presence of an individual at time $(n+1) T / 2$ in the contact process implies the existence of a path of length $n$ of closed sites, extinction of the contact process when $K$ is large follows, which completes the proof of Theorem 2.

\section{Proofs of Theorems 3 and 4 (long-range interactions)}

The proofs of both theorems are based on the analogous result for branching random walks. In this section, the branching random walk $Z_{t}$ with birth rate $\alpha$ and death rate $\Delta$ is the Markov process whose state at time $t$ consists of all functions $Z_{t}: \mathbb{R}^{d} \rightarrow \mathbb{N}$, where $Z_{t}(x)$ has to be thought of as the number of particles at point $x$. Particles die independently at rate $\Delta$, while a particle at point $x$ gives birth at rate $\alpha$ to a particle which is then sent to point $x+U$, where $U$ is uniformly distributed over the cube $(-1,1)^{d}$. Uniform random variables used to determine the locations of the offspring are chosen independently at each birth event. Note that contact processes and branching random walks differ in that births onto already occupied sites are suppressed in the former, which allows at most one particle per site, but not in the latter, which allows several particles per site. However, since the location of the offspring in the branching random walk is chosen according to a continuous random variable, the probability of the offspring being sent to any given point, or more generally any given finite set of points, is equal to 0 . In particular, starting with finitely many particles, each point is occupied by at most one particle at all times with probability 1 , provided that this property holds at time 0 , just as in the contact process. This idea will be used below in Lemmas 4 and 5 to prove weak convergence of the contact process to the branching random walk. Note also that, starting with a finite number of particles, we may identify $Z_{t}$ with the set of points that are occupied by one particle. It is a well-known result that

$$
\mathrm{P}\left(Z_{t} \neq \varnothing \text { for all } t>0 \mid Z_{0}=\{0\}\right) \neq 0 \text { if and only if } \alpha>\Delta,
$$

since the number of particles in $Z_{t}$ is a simple birth-and-death process. Note that, for both the contact process with tornadoes and the contact process with forest fires, the number of particles is dominated stochastically by the number of particles in a birth-and-death process with birth rate $\alpha$ and death rate $\Delta=1+\Lambda$, regardless of the range of the interactions. It directly follows that the critical birth parameters are bounded from below by the death rate; hence,

$$
\lim _{N \rightarrow \infty} \alpha_{\mathrm{c}}(\xi, \Lambda) \geq 1+\Lambda \quad \text { and } \quad \lim _{N \rightarrow \infty} \alpha_{\mathrm{c}}(\eta, K, \Lambda) \geq 1+\Lambda \quad \text { for all } K>0 .
$$

To establish the reverse inequalities, it suffices to prove that, for any $\alpha>1+\Lambda$, there is a positive probability of survival whenever the range of the interactions $N$ is sufficiently large. The idea is to show weak convergence of both contact processes to the branching process $Z_{t}$ and deduce the result from its analog for the branching process. Weak convergence is only true for 
the processes restricted to finite space-time regions, but combined with a rescaling argument it implies both theorems. More precisely, let $L \in \mathbb{Z}_{+}$and, for all $k \in \mathbb{Z}$, introduce the subsets

$$
I_{k}=2 k L e_{1}+[-L, L]^{d} \subset \mathbb{R}^{d} \quad \text { and } \quad J_{k}=2 k L e_{1}+(-4 L, 4 L)^{d} \subset \mathbb{R}^{d},
$$

where $e_{1}$ denotes the first unit vector in $\mathbb{Z}^{d}$. Let $\bar{Z}_{t}$ be the branching random walk modified so that offspring sent outside $J_{0}$ are killed. Again, we use a superscript to indicate the initial set. The next well-known lemma implies that, when properly rescaled in space and time, the branching random walk $Z_{t}$ dominates oriented site percolation provided that $\alpha>\Delta=1+\Lambda$.

Lemma 3. Let $T=L^{2}$ and $\alpha>1+\Lambda$. Then, for all $\varepsilon>0$, there exists $L=L(\varepsilon)$ such that

$$
\mathrm{P}\left(\operatorname{card}\left(\bar{Z}_{T} \cap I_{ \pm 1}\right) \leq L \mid \operatorname{card}\left(\bar{Z}_{0} \cap I_{0}\right) \geq L\right)<\varepsilon .
$$

Proof. This is classical and we refer the reader to [4, pp. 166-167] for a proof.

Taking $\varepsilon>0$ small such that $1-\varepsilon$ is larger than the critical value of oriented site percolation in two dimensions and then choosing $L=L(\varepsilon)$ accordingly, the previous lemma implies that there is a positive probability that the branching random walk $Z_{t}$ survives. Theorems 3 and 4 are proved following the same strategy, so it suffices to show that the contact processes modified so that offspring sent outside $J_{0}$, which we also distinguish from their unrestricted versions by adding a bar, converge weakly to the restricted branching random walk $\bar{Z}_{t}$ up to time $T$. This result is well known for the basic contact process, that is, when $\Lambda=0$, and relies on the fact that the probability of a particle being sent to a site already occupied in a given finite space-time box tends to 0 as the range of the interactions tends to $\infty$. The basic idea to extend this result in the presence of catastrophic events is to prove in addition that the probability of two particles being in the same hyperplane or in the same $K$-cube tends to 0 as the range of the interactions tends to $\infty$, as this implies that particles die individually at rate $1+\Lambda$ and never simultaneously. In particular, we also need to prove that these good properties are retained by the dynamics. In the following, we use the symbol ' $\Rightarrow$ ' to denote weak convergence in the Skorokhod space of càdlàg functions defined on the interval $[0, T]$ and with range the subsets of $\mathbb{R}^{d}$. We first deal with the contact process with tornadoes and then explain how to adapt the proof to the process with forest fires.

Definition 1. A set $A \subset \mathbb{Z}^{d} / N$ is said to be good if

$$
\pi(x) \neq \pi(y) \text { for all } x, y \in A \text { with } x \neq y,
$$

where $\pi$ denotes the orthogonal projection on the first axis.

Lemma 4. Let $A \subset I_{0} \cap \mathbb{Z}^{d} / N$ be a good set with cardinal L. As $N \rightarrow \infty$,

$$
\bar{\xi}_{t}^{A}(\alpha, \Lambda) \Rightarrow \bar{Z}_{t}^{A}(\alpha, 1+\Lambda) \quad \text { and } \quad \mathrm{P}\left(\bar{\xi}_{t}^{A}(\alpha, \Lambda) \text { is good for all } t \in(0, T)\right) \rightarrow 1
$$

Proof. First we observe that the number of offspring in the contact process produced by time $t$ is dominated by the number of particles in $Z_{t}^{A}(\alpha, 0)$, which is a pure birth process. Hence,

$$
\mathrm{E}\left|\bar{\xi}_{t}^{A}(\alpha, K, \Lambda)\right| \leq \mathrm{E}\left|Z_{t}^{A}(\alpha, 0)\right|=L \exp (\alpha t) \leq L \exp (\alpha T) \quad \text { for all } t \leq T .
$$

Then, by Markov's inequality we have

$$
\lim _{N \rightarrow \infty} \mathrm{P}\left(\left|\bar{\xi}_{t}^{A}(\alpha, K, \Lambda)\right|>N^{1 / 3} \text { for some } t \leq T\right) \leq \lim _{N \rightarrow \infty} N^{-1 / 3} L \exp (\alpha T)=0 .
$$


TABLE 1: Harris' graphical representation.

\begin{tabular}{ccc}
\hline Notation & Description & Interpretation \\
\hline$T_{n}(x)$ & Poisson process with parameter $\alpha$ & Time of a birth event \\
$U_{n}(x)$ & Uniform random variable on $(-1,1)^{d}$ & Position of the new offspring \\
$V_{n}(x)$ & Poisson process with parameter 1 & Time of an individual death \\
$W_{n}(x)$ & Poisson process with parameter $\Lambda$ & Time of a catastrophic event \\
\hline
\end{tabular}

Now, conditional on the event $E_{1}$ that the number of particles does not exceed $N^{1 / 3}$ by time $T$, at each birth, the probability that the offspring is sent to the same hyperplane as an existing particle is bounded by $N^{1 / 3}(2 N+1)^{-1}$, from which it follows that

$$
\lim _{N \rightarrow \infty} \mathrm{P}\left(E_{2} \mid E_{1}\right) \geq 1-\lim _{N \rightarrow \infty} \frac{N^{1 / 3} N^{1 / 3}}{2 N+1}=1,
$$

where $E_{2}$ is the event that no two particles' locations have the same first coordinate. The fact that the set of particles is almost surely good up to time $T$ in the limit as the range of the interactions tends to $\infty$ thus follows from the combination of (12) and (13). To prove the weak convergence of the contact process with tornadoes to the branching random walk, it suffices to show that, conditional on the event $E_{1} \cap E_{2}$, we can create a coupling such that

(i) the number of particles in both processes is the same at any time by time $T$,

(ii) each particle in one process is arbitrarily close to its counterpart in the other process provided that the range of the interactions $N$ is large.

To create such a coupling, we construct the processes from the graphical representation that consists of the independent processes introduced in Table 1. The first three processes in the table are defined for each vertex $x \in \mathbb{Z}^{d} / N$, but the last process is defined only for each vertex $x \in \mathbb{Z} / N$. Recall that both processes start with the same initial set $A$. We number the particles in $A$ from particle 1 to particle $L$ by choosing one of the possible orderings uniformly at random and, for each process, call particle number $L+i$ the particle produced at the $i$ th birth event. We claim that the contact process and branching random walk can be simultaneously constructed from the collections of independent processes of Table 1 in such a way that (i) and (ii) above hold. We simultaneously prove (i) and construct the processes, and then show that (ii) follows. Assume that (i) is true by the time $u$ of a Poisson process. We have the following alternative.

1. $u=T_{n}(x)$ for some $n$. If site $x$ is occupied by a particle of the contact process at time $u-$, say particle $i$, let $y=\pi_{N}\left(U_{n}(x)\right)$ be the (unique) vector such that

$$
y \in \frac{\mathbb{Z}^{d}}{N} \quad \text { and }\left\|U_{n}(x)-y\right\|_{\infty}<\frac{1}{2 N},
$$

and let $z \in \mathbb{R}^{d}$ denote the position of particle $i$ in the branching random walk. Then, put a particle at site $x+y$ in the contact process and a particle at point $z+U_{n}(x)$ in the branching random walk. If there is no particle at site $x$ at time $u$ - then nothing happens.

2. $u=V_{n}(x)$ for some $n$. If site $x$ is occupied by a particle of the contact process at time $u-$, say particle $i$, then particle $i$ is killed in both processes. Otherwise, nothing happens. 
3. $u=W_{n}(x)$ for some $n$. Conditional on the event $E_{2}$, there is at most one particle of the contact process in the hyperplane $\mathcal{T}_{x}$ at time $u$ - If there is one, say particle $i$, then we remove particle $i$ from both processes. Otherwise, nothing happens.

Note that rules 1-3 indicate that, conditional on the event $E_{2}$, property (i) holds at time $u$; hence, it holds up to time $T$ by a simple induction. Furthermore, the processes thus defined are indeed the contact process with tornadoes and the branching random walk, respectively. Finally, since the distance between a particle and its parent differs from at most $1 / 2 \mathrm{~N}$ between both processes, letting $y_{i}$ and $z_{i}$ denote the location of particle $i$ in the contact process and the branching random walk, respectively, adding the errors, and recalling the definitions of $E_{1}$ and $E_{2}$, we obtain

$$
\lim _{N \rightarrow \infty}\left\|y_{i}-z_{i}\right\|_{\infty} \leq \lim _{N \rightarrow \infty}\left\|y_{i-1}-z_{i-1}\right\|_{\infty}+\frac{1}{2 N} \leq \cdots \leq \lim _{N \rightarrow \infty} \frac{N^{1 / 3}}{2 N}=0
$$

almost surely on $E_{1} \cap E_{2}$, which establishes property (ii). Since, in addition, $E_{1} \cap E_{2}$ occurs almost surely in the limit as $N \rightarrow \infty$ according to (12) and (13), the result follows.

We now turn to the case of the contact process with forest fires.

Definition 2. A set $A \subset \mathbb{Z}^{d} / N$ is said to be $K$-good if

$$
\|x-y\|_{\infty}>\frac{2 K}{N} \quad \text { for all } x, y \in A \text { with } x \neq y .
$$

Lemma 5. Let $A \subset I_{0} \cap \mathbb{Z}^{d} / N$ be a $K$-good set with cardinal L. As $N \rightarrow \infty$,

$$
\bar{\eta}_{t}^{A}(\alpha, K, \Lambda) \Rightarrow \bar{Z}_{t}^{A}(\alpha, 1+\Lambda)
$$

and

$$
\mathrm{P}\left(\bar{\eta}_{t}^{A}(\alpha, K, \Lambda) \text { is } K \text {-good for all } t \in(0, T)\right) \rightarrow 1 .
$$

Proof. Observe first that the analog of (12), which is

$$
\lim _{N \rightarrow \infty} \mathrm{P}\left(\left|\bar{\eta}_{t}^{A}(\alpha, K, \Lambda)\right|>N^{1 / 3} \text { for some } t \leq T\right)=0,
$$

follows from the same comparison with a branching process as in Lemma 4 . Moreover, conditional on the event $F_{1}$ that the number of particles does not exceed $N^{1 / 3}$ by time $T$, at each birth, the probability that the offspring is sent to a $K$-cube containing at least one particle, i.e. the offspring is sent within distance $2 K / N$ of an occupied site, is less than

$$
N^{1 / 3}\left(\frac{2 N+1}{2 K+1}\right)^{-d}
$$

the maximum number of particles divided by the minimum number of $K$-cubes needed to cover an interaction neighborhood. This implies that

$$
\lim _{N \rightarrow \infty} \mathrm{P}\left(F_{2} \mid F_{1}\right) \geq 1-\lim _{N \rightarrow \infty} N^{1 / 3} N^{1 / 3}\left(\frac{2 N+1}{2 K+1}\right)^{-d}=1,
$$

where $F_{2}$ is the event that each $K$-cube has at most one particle by time $T$. Hence, the set of particles is almost surely good up to time $T$ in the limit as $N$ tends to $\infty$. To prove weak 
convergence on the event $F_{1} \cap F_{2}$, we couple the contact process and the branching random walk by ordering the particles as in Lemma 4 and by using the graphical representation of Table 1 , except that the process $W .(x)$ now has intensity $\Lambda(2 K+1)^{-d}$ and is defined for all $x \in \mathbb{Z}^{d} / N$, and that rule 3 in the proof of Lemma 4 is replaced by the following rule.

$3^{\prime}$. If $u=W_{n}(x)$ for some $n$ then, conditional on the event $F_{2}$, there is at most one particle of the contact process in the $K$-cube with center $x$ at time $u$-. If there is one, say particle $i$, then we remove particle $i$ from both processes. Otherwise, nothing happens.

The fact that the number of particles is the same in both processes follows from the conditioning on the event $F_{2}$, which implies that each forest fire kills at most one particle. Finally, the same calculation as in Lemma 4 implies that the distance between each particle in one process and its counterpart in the other process tends to 0 as $N$ tends to $\infty$.

As previously explained, Theorem 3 follows from the combination of Lemmas 3 and 4, while Theorem 4 follows from the combination of Lemmas 3 and 5.

\section{Acknowledgement}

The author would like to thank an anonymous referee for pointing out several mistakes in a preliminary version of this work.

\section{References}

[1] Belhadji, L. and Lanchier, N. (2006). Individual versus cluster recoveries within a spatially structured population. Ann. Appl. Prob. 16, 403-422.

[2] Belhadj, L. And Lanchier, N. (2008). Two-scale contact process and effects of habitat fragmentation on metapopulations. Markov Process. Relat. Fields 14, 487-514.

[3] Bezuidenhout, C. And Grimmett, G. (1990). The critical contact process dies out. Ann. Prob. 18, $1462-1482$.

[4] Durrett, R. (1995). Ten lectures on particle systems. In Lectures on Probability Theory (Saint-Flour, 1993; Lecture Notes in Math. 1608), Springer, Berlin, pp. 97-201.

[5] Durrett, R. and Remenik, D. (2009). Chaos in a spatial epidemic model. Ann. Appl. Prob. 19, 1656-1685.

[6] Harris, T. E. (1974). Contact interactions on a lattice. Ann. Prob. 2, 969-988.

[7] Kang, H.-C., Krone, S. M. and Neuhauser, C. (1995). Stepping-stone models with extinction and recolonization. Ann. Appl. Prob. 5, 1025-1060.

[8] Liggett, T. M. (1999). Stochastic Interacting Systems: Contact, Voter and Exclusion Processes Wissenschaften (Fundamental Principles Math. Sci. 324), Springer, Berlin.

[9] Liggett, T. M., Schinazi, R. B. And Schweinsberg, J. (2008). A contact process with mutations on a tree. Stoch. Process. Appl. 118, 319-332.

[10] Richardson, D. (1973). Random growth in a tessellation. Proc. Camb. Phil. Soc. 74, 515-528.

[11] Schinazi, R. B. (2002). On the role of social clusters in the transmission of infectious diseases. Theoret. Pop. Biol. 61, 163-169.

[12] Schinazi, R. B. (2005). Mass extinctions: an alternative to the Allee effect. Ann. Appl. Prob. 15, 984-991.

[13] SchinAzI, R. B. AND Schweinsberg, J. (2008). Spatial and non-spatial stochastic models for immune response. Markov Process. Relat. Fields 14, 255-276.

[14] Van den Berg, J., Grimmett, G. R. and Schinazi, R. B. (1998). Dependent random graphs and spatial epidemics. Ann. Appl. Prob. 8, 317-336. 